\title{
Study of oxidative stress and antioxidant status in ascitic patients with ovarian cancer in comparison to liver cirrhosis patients
}

\begin{abstract}
Background: The pathological accumulation of abdominal fluid (ascites) is due to multiple causes often associated either with peritoneal diseases or non-peritoneal diseases. Oxidative stress is caused by free radicals leading to oxidative destruction of polyunsaturated fatty acids constitutive of cellular membrane. The present study was conducted to assess the oxidative stress and antioxidant status in ascitic patients with liver cirrhosis in comparison to patients with ovarian carcinoma. Serum and ascitic fluid concentrations of malondialdehyde (MDA), Superoxide dismutase (SOD/ gm.protein) and Ceruloplasmin (CP) was assessed in 375 ascitic patients, among them 145 patients were females with ovarian cancer (Age 19-84yrs; mean age 49.85 11.75 ); 230 were liver cirrhosis patients (178 males and 52 females age 20-75yrs; mean age $46.59 \pm 10.82)$ and 150 subjects were healthy controls ( 91 males and 59 females age 24-65yrs; mean age 43.31 \pm 8.51 ). MDA and SOD was estimated by manual methods; Ceruloplasmin(CP) was measured by immunoturbidometric assay using commercial kit. Significant increased levels of serum and ascitic fluid MDA and CP and decreased levels of SOD was observed in ovarian cancer patients when compared to that of liver cirrhosis and control group $(\mathrm{p}<0.001)$. Significant increased levels of serum MDA and decreased levels of CP and SOD was observed in liver cirrhosis patients when compared to that of control $(\mathrm{p}<0.001)$.
\end{abstract}

Conclusion: It could be inferred from the experiments conducted that MDA and $\mathrm{CP}$ increased in ovarian cancer patients while there was a reduction in SOD. MDA showed the same trends in liver cirhossis patients however CP and SOD showed decreased levels in liver cirrhosis patients. Thus it is possible that CP could seem as a biomarker for screening of ovarian carcinoma. The financial implication for screening also would be affordable to the common man. Thus the most salient feature of this communication is identification of a suitable biomarker for screening ovarian cancer which is affordable. CP levels thus could prove a valuable assay for the screening, diagnosis and monitoring of ovarian cancer.

Keywords: ascites, liver cirrhosis, ovarian cancer, oxidative stress, Ceruloplasmin, biomarker, antioxidants
Volume 6 Issue I - 2017

\author{
Khaleel Pasha,' Gopal Reddy M,'² Ramesh \\ Kumar B,' Qamar Ayesha,' Srinivasulu M, ${ }^{3}$ \\ Suseela K, ${ }^{4}$ Gita Sharma, ${ }^{5}$ Livy Alex ${ }^{6}$ \\ 'Department of Gastroenterology, Osmania General Hospital, \\ India \\ ${ }^{2}$ Department of Biochemistry, Malla Reddy Narayana \\ Multispeciality Hospital Suraram, India \\ ${ }^{3}$ Department of Surgical Oncology, MNJ Institute of Oncology \\ and Regional Centre, India \\ ${ }^{4}$ Department of Pathology, Basavatarakam Indo-American \\ Cancer Hospital \& Research Institute, India \\ ${ }^{5}$ Tapadia Diagnostics Centre, India \\ ${ }^{6}$ SLS Cell Cure Technologies Pvt Ltd, India
}

Correspondence: Livy Alex, Technical Director, SLS Cell Cure Technologies Pvt Ltd., I03,Prabhat Apartments, Street no I I,East Maredpally, Hyderabad-500026, India, Tel +919652874747, 040277344 I5, Fax 040 77344 I5, Email drlivys@yahoo.com

Received: June 18, 2017 | Published: September 07, 2017

\section{Introduction}

The pathological accumulation of abdominal fluid (ascites) is due to multiple causes often associated either with peritoneal and non-peritoneal diseases. In the process of ascites formation there is increased vascular permeability, decreased lymphatic resorption and gross change in the concentrations of functional proteins and metabolites. Liver cirrhosis is a chronic disorder in which ascites develop in advanced stages of the disease, where as in ovarian cancer, most of the cases develop ascites in the early stages after the onset of disease. Among malignant ascites, ovarian cancers are the most common primary tumors and sixth most common malignant neoplasm in women with highest mortality rate. ${ }^{1}$ Oxidative stress is caused by free radicals leading to oxidative destruction of poly unsaturated fatty acids constitutive of cellular membrane and give rise to lipid peroxides which are unstable and decompose to form reactive carbonyl compounds. Among them malondialdehyde (MDA) is the most abundant, and it readily combines with several functional groups on proteins and forms DNA-MDA adducts, which are highly mutagenic, cytotoxic, co-carcinogenic and acts as a tumor promoter. $^{2-5}$ Oxidative stress induces cancer among the biological molecules and plays an important role in the pathogenesis of liver disease and other hepatic alterations. ${ }^{6}$ Intestinal permeability is increased in patients with cirrhosis and oxygen free radicals play an important role in gut epithelial damage. Hence measurement of MDA is widely used as an indicator of oxidative stress. The antioxidant enzyme superoxide dismutase (SOD) is widely distributed in the cells and present in higher levels in erythrocytes, ${ }^{7}$ and it plays an important role in scavenging and protects cells against superoxide radical by dismutation of the highly reactive superoxide anion to oxygen and less reactive oxygen species hydrogen peroxide. ${ }^{8}$ The low levels of SOD may be due to increased utilization of scavenger lipid peroxides. The imbalance between the free radicals and antioxidants results in oxidative stress. Ceruloplasmin is a copper binding protein which is increased in several carcinomas.

\section{Materials and methods}

\section{Informed consent}

This study was approved by the Institutional Ethics Committee 
of the hospital. Informed oral consent was obtained from each participant, after explaining the purpose of this study in their own language, before obtaining the blood sample.

\section{Study Group}

A total number of 375 ascitic patient's venous blood and ascitic fluid samples were collected from confirmed cases of liver cirrhosis and ovarian cancer patients who were devoid of renal, Pancreatic and tuberculosis from Liver care unit of Osmania General Hospital, MNJ Institute of Oncology and Regional Cancer Centre and Basavatarakam Indo-American Cancer Hospital \& Research Institute Hyderabad, over a period of two years from May 2014 to June 2016. Among them 145 patients were females with ovarian cancer of age 19-84yrs (mean age 49.85 \pm 11.75 ); 230 patients with liver cirrhosis(178 males and 52 females of age 20-75yrs), (mean age 46.59 \pm 10.82 ) and 150 blood samples were collected from healthy controls of age 24-65yrs, 91 males and 59 females (mean age 43.31 18.51 ). Inclusion criteria: Patient diagnosed by history, clinical examination and laboratory investigations to have ovarian cancer and liver cirrhosis without any complications falling in the age group of 19-84 years was included in the study. Apparently healthy people who are age and sex matched with the sample group were used as controls for the study.

\section{Exclusion criteria}

Patients with, renal failure, pancreatitis and Tuberculosis were excluded from the study.

\section{Sample collection}

Serum was separated immediately and stored along with the corresponding ascitic fluid at $-70{ }^{\circ} \mathrm{C}$ for analysis of proposed parameters.

\section{Statistical analysis}

Data are expressed as Mean \pm SD. The statistical significance was determined by one-way (ANOVA) followed by post hoc test by LSD (least significance difference) for significance. A $p$ value $<0.05$ was considered statistically significant.

The patient's history was recorded in the specific proforma approved by the Ethics Scientific Committee of Osmania Medical College and Basavatarakam Indo-American Cancer Hospital \& Research Institute Hyderabad.

\section{Biochemical estimations}

MDA was measured in serum and ascitic fluid on the same day of collection by the manual method of Brid and Draper 1984 , using thiobarbituric acid reaction. SOD/gm. protein was measured by inhibition of auto-oxidation of adrenaline of Hara Misra and Irwin Fridovich 1972. Ceruloplasmin was estimated by immunoturbidometric assay by using commercial kit from Agappe diagnostics Ltd.

\section{Results}

The results in the serum samples of two groups were analyzed separately and compared with healthy controls, whereas in the ascitic fluid results were compared among the groups. The mean serum concentration of MDA and CP was significantly increased in ovarian cancer patients when compared to that of liver cirrhosis and control groups $(\mathrm{p}<0.001)(1055.52 \pm 97.37$ vs $556.15 \pm 90.59 ; 392.0 \pm 36.63)$; $(67.04 \pm 13.10$ vs $28.68 \pm 6.82 ; 35.97 \pm 7.15)$ respectively. Significant increased levels of serum MDA and decreased levels of serum CP were found in liver cirrhosis patients when compared to that of controls $(\mathrm{p}<0.001)(556.15 \pm 90.59$ vs $392.0 \pm 36.63) ;(28.68 \pm 6.82$ vs $35.97 \pm 7.15$ ) respectively. The mean serum concentration of SOD was grossly decreased in both the groups when compared to that of controls $(p<0.001)(40.70 \pm 10.53 ; 44.03 \pm 11.76$ vs $64.93 \pm 9.46)$ respectively. The mean ascitic fluid concentration of MDA and CP was increased significantly in ovarian cancer when compared to that of liver cirrhosis $(\mathrm{p}<0.001)(931.40 \pm 88.11 ; 33.90 \pm 7.88$ vs $449.57 \pm 90.35 ; 12.20 \pm 5.28)$ respectively. The mean concentration of SOD in ascetic fluid was grossly decreased in ovarian cancer patients when compared with that of liver cirrhosis patients $(\mathrm{p}<0.001)(38.92 \pm 9.83$ vs $183.17 \pm 72.44)$. The results are depicted in Tables $1 \& 2$. Significance of $p$ value comparison among groups is shown in Figures $1 \& 2$.

Table I Serum concentrations Malondialdehyde (MDA) Ceruloplasmin (CP) Superoxide dismutase (SOD/gm.protein) in ovarian cancer, liver cirrhosis, and control group (mean $\pm \mathrm{SD})$

\begin{tabular}{llll}
\hline Parameters & & N & Mean \pm SD \\
\hline \multirow{3}{*}{ MDA (nmol/dl) } & Ovarian cancer & 145 & $1055.52 \pm 97.37$ \\
& Liver cirrhosis & 230 & $556.15 \pm 90.59$ \\
& Control & 150 & $392.0 \pm 36.63$ \\
& Ovarian cancer & 145 & $40.70 \pm 10.53$ \\
SOD/gm Protein & Liver cirrhosis & 230 & $44.03 \pm 11.76$ \\
& Control & 150 & $64.93 \pm 9.46$ \\
& Ovarian cancer & 145 & $67.04 \pm 13.10$ \\
Ceruloplasmin (mg/d) & Liver cirrhosis & 230 & $28.68 \pm 6.82$ \\
& Control & 150 & $35.97 \pm 7.15$ \\
\hline
\end{tabular}

Table 2 Ascitic fluid concentrations Malondialdehyde (MDA), Superoxide dismutase (SOD), Ceruloplasmin (CP) in Liver cirrhosis and Ovarian cancer and control group, (mean $\pm S D$ ); OC \& LCVs C indicates ovarian cancer and liver cirrhosis vs control

\begin{tabular}{llll}
\hline Parameters & & N & Mean \pm SD \\
\hline \multirow{2}{*}{ MDA $(\mathrm{nmol} / \mathrm{dl})$} & Ovarian cancer & 145 & $931.40 \pm 88.1 \mathrm{I}$ \\
& Liver cirrhosis & 230 & $449.57 \pm 90.35$ \\
SOD/gm Protein & Ovarian cancer & 145 & $38.92 \pm 9.83$ \\
& Liver cirrhosis & 230 & $183.17 \pm 72.44$ \\
Ceruloplasmin $(\mathrm{mg} / \mathrm{dl})$ & Ovarian cancer & 145 & $33.90 \pm 7.88$ \\
& Liver cirrhosis & 230 & $12.20 \pm 5.28$ \\
\hline
\end{tabular}

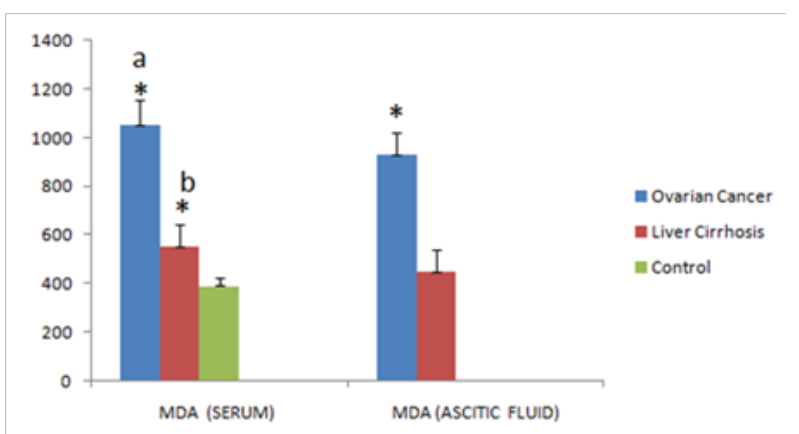

Figure I Significance of serum and ascitic fluid concentrations of Malondialdehyde (MDA) in ovarian cancer, liver cirrhosis and control group. $a^{*}$ indicates $p<0.001$ ovarian cancer vs liver cirrhosis \& control; $b^{*} p<0.00$ I liver cirrhosis vs control; $* p<0.00 \mathrm{I}$ indicates ascitic fluid from ovarian cancer vs liver cirrhosis. 


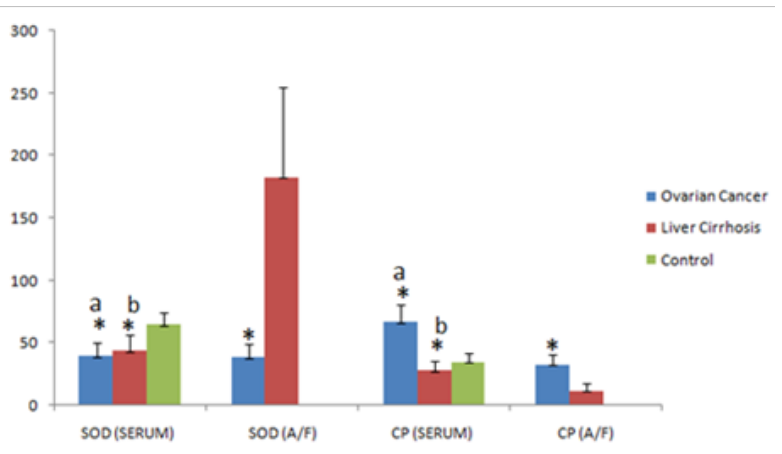

Figure 2 Significance of serum and ascitic fluid concentrations Superoxide dismutase (SOD) and Ceruloplasmin (CP) in ovarian cancer, liver cirrhosis and control group, (mean $\pm \mathrm{SD})$; $\mathrm{a}^{*}$ indicates $p<0.00 \mathrm{I}$ ovarian cancer vs liver cirrhosis \& control; $b * p<0.00$ I liver cirrhosis vs control; $* p<0.00$ I indicates ascitic fluid ovarian cancer vs liver cirrhosis.

\section{Discussion}

In the present study we have observed significant increased levels of serum MDA, CP and decreased levels of SOD in ovarian cancer patients when compared to that of liver cirrhosis and controls. These finding has been replicated in many studies.

Sanjyothi et al., ${ }^{9}$ found elevated levels of serum MDA and decreased levels of erythrocyte superoxide dismutase (SOD) in ovarian cancer patients when compared to that of controls. They suggested increased levels of MDA may be due to excessive oxidative stress caused by incessant ovulation or epithelial inflammation initiators such as talc and asbestos. The decreased levels of SOD may be due to increased utilization to scavenge lipid peroxides as well as their sequestration by tumor cells. Similar findings were also found in the study of Hathama et al. ${ }^{4}$ In another study by Talat et al., ${ }^{10}$ they found significantly increased levels of MDA in the serum samples of ovarian cancer patients when compared to that of controls. Similar findings were also found in serum samples of other cancers patients. ${ }^{11}$ Hietanen et al., ${ }^{12}$ concluded that oxidative stress may be associated with malignant disease.

There are many studies showing that patients with malignancies have higher incidence of reactive oxygen species (ROS) and lower concentration of antioxidants as the spread of malignancy increases. ${ }^{13}$ It is suggested that increased ROS leads to an increase in DNA mutation as well as increased immuno-suppression. ${ }^{14,15}$

According to the study of Alessandra Buico et al., ${ }^{16}$ increased levels of serum ceruloplasmin in the ovarian cancer patients often accompany neoplastic processes and may be caused by interleukin-1 and tumor necrosis factor released by macrophages. Increased concentrations of ceruloplasmin were also reported in several carcinomas. ${ }^{17,18}$ The results of this study are in concordance with the present study.

The present study also showed statistically significant increased levels of serum MDA, decreased levels of SOD and CP in patients with liver cirrhosis. Many studies have shown increased levels of serum MDA and decreased levels of antioxidants in patients with liver cirrhosis.

In the study of Hulya Aksoy et al., ${ }^{19}$ they found increased levels of serum MDA and decreased levels of ceruloplasmin in liver cirrhosis patients. They suggested increased level of MDA is due to increased lipid peroxidation and antioxidant levels were typically depleted in liver cirrhosis patients which has been reported in various liver diseases. ${ }^{20,21}$ Our data confirms the presence of an increased oxidative stress in liver cirrhosis patients and suggested more oxidative stress results from liver failure or inflammation as liver disease progresses.

Suresh Chary \& Madhur Gupta ${ }^{22}$ also found similar findings of increased levels of MDA and decreased levels of SOD in alcoholic liver cirrhosis patients when compared to controls. They suggested increased levels of MDA may be due to ethanol toxicity on the liver as ethanol impairs the hepatic antioxidant potential. ${ }^{22}$ It is suggested that free radical intermediate and disturbances of iron metabolism reported earlier in human alcoholics might be responsible for causing oxidative damage. ${ }^{23,24}$

Seren Ozenirler et al., ${ }^{25}$ found increased levels of serum SOD and MDA levels in patients with liver cirrhosis when compared to that of controls. Our study showed decreased levels of SOD in serum and increased levels of SOD in ascitic fluid in patients with liver cirrhosis, which is positively correlated with the previous studies of Makarenko et al. ${ }^{26}$ Zhurkin et al. ${ }^{27}$ They suggested cirrhotic patients showed an adaptive reaction directed to the prevention of lipid peroxidation and its injurious effect on the cellular structures. Shaden M Hanafy et al., ${ }^{28}$ found decreased levels of serum SOD in cirrhosis of liver patients when compared to that of controls, while it significantly increased after the treatment.

Prakash \& Joshi found increased levels of serum MDA in liver cirrhosis patients when compared to that of healthy controls, where as vitamin $\mathrm{E}$ and $\mathrm{C}$ levels were significantly decreased which indicates that the breakdown of antioxidant defense could be the cause for the development and progression of liver cirrhosis. Increased levels of ascitic fluid SOD in liver cirrhosis patients may indicate the compensatory mechanisms to overcome the oxidative stress in the liver cirrhosis patients. ${ }^{29,30}$

\section{Funding information}

This study was not supported by any grants, and the finance was borne by the first author himself.

\section{Acknowledgements}

We would like to thank the Directors of MNJ institute of Oncology and Regional Cancer centre and Basavatarakam Indo-American cancer Hospital and Research Institute, Hyderabad, for providing the materials for this study.

\section{Conflict of interest statement}

All co-authors of the manuscript have no conflict of interest and have nothing to disclose.

\section{References}

1. Lengyel E. Ovarian cancer development and metastasis. Am J Pathol. 2010;177(3):1053-6410.

2. Kang DH. Oxadative stress, DNA damage and breast cancer. AACN Clin Issues. 2002;13(4):540-549.

3. Ahmed MI, Fayed ST, Hossien H, et al. Lipid peroxidation and antioxidant status in D.J human cervical carcinoma. Dis Markers. 1999;15(4):283-291.

4. Hathama R, Hasan, Shatha A, et al. Serum lipid peroxidation and trace elements levels in ovarian cancer patients before and after cisplatin and doxorubicin chemotherapy. Journal of college education. 2011:5.

5. Srivastava S, Natu SM, Gupta A, et al. Lipid peroxidation and antioxidant in different stages of cervical cancer Prognostic significance. Indian $J$ cancer. 2009;46(4):297-302. 
6. Groszmann RJ. Hyperdynamic circulation of liver disease 40years later: Pathophysiology and clinical consequences. Hepatology. 1994;20(5):1359-1363.

7. Speranza MJ, Bagley AC, Lynch RE. Cells Enriched for catalase are sensitized to the Toxicities of Bleomycin, Adriamycin, and Paraquat. $J$ Bio Chem. 1993;268(25):1939-1943.

8. McCord JM. The Evolution of Free Radicals and oxidative stress. Am J Med. 2000;108(8):652-659.

9. Sanjyothi Bandebuche, Melinkeri RR. Oxidative stress and antioxidant status in patient of ovarian cancer. Biomedical Research. 2011;22(2):193197.

10. Talat TK. Basicsciences-Dentistry college-Babylan Uni. The relationship between serum Copper and the Copper/Zinc ratio with malondialdehyde in patients with ovary cancer. 2010. p. 516-524.

11. Punnonen K, Ahotupa M, Asaishi K, et al. Antioxidant enzyme activities and oxidative stress in human cancer. $J$ Cancer Res Clin Oncol. 1994;120(6):374-377.

12. Hietanen E, Bartsch H, Béréziat JC, et al. Diet and oxidative stress in colon and prostate cancer patients: A case-control study. Eur J Clinical. 1994;48(8):575-586.

13. Khanzode SS, Muddershwar MG, Khanzode SD, et al. Antioxidant enzyme and lipid peroxidation in different stages of breast cancer. Free Radic Res. 2004;38(1):81-85.

14. Hathama R, Hasan, Shatha A, et al. Serum lipid peroxidation and trace elements levels in ovarian cancer patients before and after cisplatin and doxorubicin chemotherapy. Journal of college education. 2011:5.

15. Borek C. Dietary antioxidants and human cancer. Integr Cancer Ther. 2004;3(4):333-341.

16. Buico A, Cassino C, Ravera M, et al. Oxidative Stress and Total antioxidant Capacity in human plasma. Redox Report. 2009;14(3):125131

17. Zowczak M, Iskra M, Paszkowski J, et al. Oxidase activity of ceruloplasmin and concentrations of copper and zinc in serum of cancer patients. J Trace Elem Med Biol. 2001;15(2-3):193-196.

18. Nayak SB, Bhat VR, Mayya SS. Serum copper, ceruloplasmin and Thiobarbituric acid substance status in patients with ovarian cancer. Indian J Physiol Pharmacol. 2004;48(4):486-488.
19. Hulya Aksoy, Ahhmet Kiziltunc, Yilmaz Aksosy, et al. Determination of pH, Fibronectin, cholesterol, Lactate dehyderagenase and sialic acid in the differentiation of non malignant and malignant asites. Tr J Medical Sciences. 1997;28:549-553.

20. Shaw S, Rubin KP, Lieber CS. Depressed hepatic glutathione and increased diene conjugates in alcoholic liver disease. Evidence of lipid peroxidation. Diag DIs Sci. 1983;28(7):585-589.

21. Mazzanti R, Moscarella S, Bensi G, et al. Hepatic lipid peroxidation and aldehyde dehrogenase activity in alcoholic and non-alcoholic liver disease. Alcohol. 1989;24(2):121-128.

22. Suresh Chari, Madhu Gupta. Status of blood antioxidant enzyme in alcoholic cirrhosis. Indian Journal of Physiology and pharmacology. 2003;47(3):343-346.

23. Rouach H, Fataccioli V, Gentil M, et al. Effects of chronic ethanol feeding on lipid peroxidation and protein oxidation in relation to liver pathology. Hepatology. 1997;25(2):351-355.

24. Clot $\mathrm{P}$, Tabone $\mathrm{M}$, Aricò $\mathrm{S}$, et al. Monitoring oxidative damage in patients with liver cirrhosis and different daily alcohol intake. Gut. 1994;35(11):1637-1643.

25. Ozenirler S, Sancak B, Coskun U. Serum and ascitic fluid superoxide dismutase and malondialdehyde levels in patients with cirrhosis. Biomark Insights. 2008;3:141-145.

26. Makarenko EV, Kozlowski IV. The Erythrocytes antioxidant system in chronic liver disease. Ter Arkh. 2000;61:115-118.

27. Zhurkin AT, Dubinima EE, Koryagin VN. Immunological indicators and superoxide desmutase activity in the erythrocytes of patients with viral hepatitis. Ter Arkh. 1989;61(2):58-60.

28. Hulya Aksoy, Mehmet Koruk, Faith Akcay. The relationship between serum Malondialdehyde and ceruloplasmin in chronic liver disease. Turk J Biochem. 2003;28(2):32-34.

29. Malmberg KJ, Lenkei R, Petersson M, et al. A short-term dietary supplementation of high doses of vitamin $\mathrm{E}$ increases $\mathrm{T}$ helper-1cytokine production in the patients with advanced colorectal cancer. Clin Cancer Res. 2002;8(6):1772-1778.

30. Pejić S, Kasapović J, Todorović A, et al. Lipid peroxidation and antioxidant status in blood of patients with uterine myoma, endometrial polypus, Hyper plastic and malignant endometrium. Biol Res. 2006;39(4):619-629. 